\title{
GIST: avaliações morfológica e imuno-histoquímica do prognóstico
}

Primeira submissão em 26/01/09 Última submissão em 27/01/09 Aceito para publicação em 20/02/09 Publicado em 20/02/09

\author{
GIST: morphological and immunohistochemical prognostic evaluation
}

Rodrigo Panno Basilio de Oliveira'; Vera Lucia Pannain²; Pedro Portari3; Antonio Carlos Iglesias ${ }^{4}$;

Carlos Alberto Basilio-de-Oliveira ${ }^{5}$

unitermos
Tumor estromal
gastrointestinal
Morfologia
Imuno-histoquímica
Prognóstico

\section{resumo}

Introdução: os tumores estromais gastrointestinais possuem amplo espectro biológico, variando desde lesões de comportamento benigno até aquelas de caráter maligno, capazes de ampla disseminação e frequentes metástases viscerais. Atualmente, o prognóstico está baseado num escore, denominado grau de risco. Entretanto, este sistema apresenta falhas, com tumores classificados como de riscos intermediário e baixo associados ao desenvolvimento de metástases. Desta forma, são necessários estudos que visem ao aprimoramento desse sistema de classificação, destacando-se nesse campo, nos últimos anos, o índice de proliferação celular que tem mostrado valor prognóstico na predição da agressividade tumoral. Objetivos: analisar critérios morfológicos (tamanho macroscópico, topografia do tumor, índice mitótico, necrose, subtipo histológico), verificar o grau de risco e pesquisar a aplicabilidade dos marcadores imunohistoquímicos (actina músculo-específico, proteína S-100, Ki67 e p16ink4a) como fatores prognósticos do tumor estromal gastrointestinal (GIST). Resultados: a análise univariada mostrou significância com tumores maiores que $5 \mathrm{~cm}$, número de mitoses maior que 5/50 CGA, presença de necrose, de grau de risco alto e índice de proliferação celular (Ki67) maior que $5 \%$ com relação à redução da sobrevida global dos pacientes $(p=0,017 ; 0,01 ; 0,001 ; 0,016 ;<0,001$ respectivamente). Os outros fatores analisados (subtipo histológico, imunofenótipo e p16 ${ }^{\text {ink4a }}$ ) não mostraram significância. Conclusão: o grau de risco, o tamanho tumoral, o índice mitótico e a presença de necrose corroboram evidências prévias da sua utilização, como fatores morfológicos prognósticos e o emprego do índice de proliferação celular (Ki67) associado ao grau de risco para melhor esclarecimento do comportamento biológico dos GIST.

\section{abstract}

Introduction: Gastrointestinal stromal tumors (GIST) have a wide biological spectrum, ranging from benign to malignant lesions, which are prone to wide spread and frequent visceral metastasis. Currently, the prognosis is based on a score system known as risk level. However, this system has some drawbacks. For instance, tumors classified as low or intermediate risk may be associated with the development of metastasis. Therefore, studies are required to improve this classification system and incorporate recent developments such as cellular proliferation index, which has shown prognostic value in the prediction of tumor aggressiveness. Objectives: To analyze morphological criteria (macroscopic size, tumor topography, mitotic index, necrosis, histological subtype), observe risk and investigate the usefulness of immunohistochemical markers (muscle-specific actin, S-100 protein, Ki67 and p16ink4a) as prognostic markers of GIST. Results: Univariate analysis showed that a reduced global survival was significantly associated with tumor size greater than $5 \mathrm{~cm}$, mitotic index greater than 5/50 CGA, presence of necrosis, a high risk level, and a cellular proliferation index (Ki67) higher than 5\% on the reduction of overall survivel of patients $(p=0.017,0.010,0.001,0.016$ and 0.0005 , respectively). Other factors such as histological subtype, immunophenotype and p1 6ink4a were not significant. Conclusion: According to our data, risk level, tumor size, mitotic index and the presence of necrosis stood as morphological predictors of reduced survival, which underpins previous evidence of their application. The cellular proliferation marker Ki67 associated with risk level also proved to be a useful predictor of tumor aggressiveness.

\section{key words}

Gastrointestinal stromal tumor

Morphology

Immunohistochemical

Prognostic

1. Professor assistente da disciplina de Anatomia Patológica da Escola de Medicina e Cirurgia da Universidade do Rio de Janeiro (EMC/UNI-RIO).

2. Professora adjunta do Departamento de Patologia da Universidade Federal do Rio de Janeiro (UFR)).

3. Professor adjunto do Departamento de Cirurgia Ceral e Especializada da UNI-RIO.

4. Professor titular da disciplina de Clínica Cirúrgica A da EMC/UNI-RIO.

5. Professor titular da disciplina de Anatomia Patológica da EMC/UNI-RIO.

0 presente estudo foi realizado nos hospitais universitários Caffrée e Guinle (HUGC) e Clementino Fraga Filho (HUCFF), fazendo parte dos pré-requisitos para obtenção do título de mestre em Anatomia Patológica na Faculdade de Medicina da UFRJ, tendo como título "Tumores estromais gastrointestinais. Contribuição morfológica e imuno-histoquímica para avaliação do prognóstico" e sendo aprovado pelo comitê de ética em pesquisa do HUCFF com o número 079/05. 


\section{Introdução}

Atualmente, o tumor estromal gastrointestinal (GIST) é considerado a neoplasia mesenquimal mais comum do tubo gastrointestinal $(\mathrm{TCl})^{(18,30)}$. Entre $10 \%$ e $30 \%$ dessas neoplasias evidenciam comportamento agressivo, tendo como principais sítios metastáticos o fígado e a cavidade peritoneal ${ }^{(5,17)}$. Originam-se a partir das células intersticiais de Cajal, que constituem verdadeiro marca-passo celular do tubo disgestivo ${ }^{(14,24)}$. Esta célula, de natureza mesenquimal, apresenta em sua superfície receptor tirosina-quinase subclasse III (Kit), codificado pelo proto-oncogene c-Kit(36), que, quando ativado, altera o receptor tirosina-quinase, principalmente no seu domínio justamembrana, o que corresponde à mutação do éxon $11^{(12)}$. Outros locais de mutação podem estar envolvidos, como o éxons 9,13 e $17^{(1,12,13,27)}$. O seu diagnóstico está baseado na expressão imuno-histoquímica do anticorpo CD $117^{(9)}$.

A principal localização do tumor estromal de células de Cajal é no estômago (cerca de 60\%), seguidos do intestino delgado (30\%), cólons e reto (5\%) e esôfago (5\%) ${ }^{(33)}$. Em algumas ocasiões, os GISTs podem se desenvolver fora do trato gastrintestinal ( $\mathrm{TGl})$, como no mesentério, no omento ou no retroperitônio ${ }^{(8,23)}$. Ao exame macroscópico, variam de nódulos com poucos centímetros até grandes massas sólidas com áreas de necrose (Figuras 1 e 2) 2 $^{(9)}$. À luz da microscopia apresenta-se em três clássicos subtipos: fusiforme, epitelioide e misto (fusiforme e epitelioide). Destes, o fusiforme é o mais frequente e corresponde a cerca de $70 \%$ dos $\operatorname{casos}^{(9)}$.

O prognóstico das neoplasias estromais gastrointestinais ainda é controverso e está baseado num escore ${ }^{(9)}$, que fornece o grau de risco de metástase e/ou recidiva,

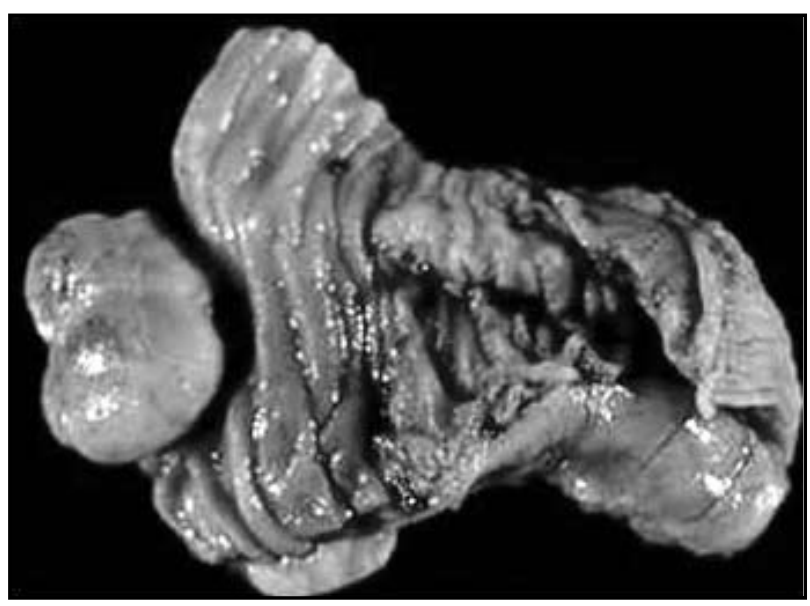

Figura 1 - GIST gástrico de pequenas dimensões

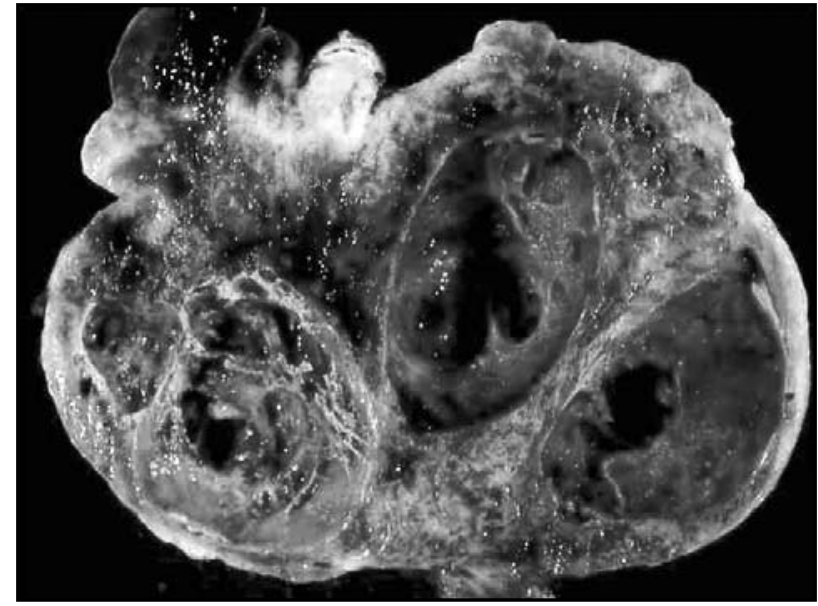

Figura 2 - Massa tumoral aderida a segmento colônico, a qual evidencia extensas áreas de necrose

baseando-se no índice mitótico e no tamanho da neoplasia. Deve-se ressaltar, todavia, que o assunto está longe de ser resolvido, visto que existem neoplasias consideradas como de baixo risco que mostram comportamento biológico agressivo e com potencial metastático ${ }^{(9,26)}$. Na busca de possíveis indicadores que melhor retratem o prognóstico desta neoplasia, analisamos a contribuição dos marcadores imuno-histoquímicos p16 $6^{\text {inkaa }}$ (proteína reguladora do ciclo celular), do Ki67 (índice de proliferação celular), do grau de risco, do imunofenótipo e dos achados histológicos, como: necrose, índice mitótico e subtipo.

\section{Pacientes e métodos}

\section{Dados clínicopatológicos}

O estudo foi transversal, retrospectivo, em 42 casos de GIST, com expressão imuno-histoquímica fortemente positiva para o anticorpo anti-CD117. Estes corresponderam a tumores completamente ressecados entre janeiro de 1982 e fevereiro de 2005, no Hospital Universitário Clementino Fraga Filho da Universidade Federal do Rio de Janeiro (HUCFF/UFRI) e no Hospital Universitário Gaffrée e Guinle da Universidade do Rio de Janeiro (HUGG/UNI-RIO). Nesta amostra, os 42 pacientes não tinham evidência de doença disseminada no ato operatório, e os dados, como sexo, idade, tamanho macroscópico e evolução clínica, foram obtidos dos prontuários médicos. O tempo de evolução foi contado a partir da data da cirurgia até o último contato do paciente, com seguimento de um a 195 meses. O prognóstico foi dividido em: paciente vivo e sem recorrência da doença; vivo e com recorrência da doença; e os casos de óbito pela doença. 
Quanto à morfologia foram avaliados o índice mitótico, o subtipo histológico, a necrose e o grau de risco de acordo com o consenso (Tabela) ${ }^{(9)}$.

\begin{tabular}{lcc} 
Tabela & \multicolumn{2}{c}{ Consenso prognóstico do GIST(9) } \\
\hline Grau de risco & $\begin{array}{c}\text { Tamanho } \\
\text { macroscópio (cm) }\end{array}$ & $\begin{array}{c}\text { Índice mitótico } \\
(50 \mathrm{CGA})\end{array}$ \\
Baixíssimo & $<2$ & $<5$ \\
Baixo & $2-5$ & $<5$ \\
& $<5$ & $6-10$ \\
Intermediário & $5-10$ & $<5$ \\
& $>5$ & $>5$ \\
Alto & $>10$ & qualquer índice \\
& qualquer tamanho & $>10$
\end{tabular}

Foram utilizados os anticorpos monoclonais Dako: anti-CD117 (diluição: 1:100, código A4502), anti-Ki67 (diluição:1:250, código M7240), antiproteína S-100 (diluição:1:1.000 código Z0311), antiactina músculo-específico (diluição:1:250, código M0635) e p16 ink4a (diluição 1:20) K5334 pronto para uso. As lâminas foram mergulhadas em tampão de ácido etilenodiaminotetracético (EDTA)/TRIS com $\mathrm{pH}=9$, em banho-maria, à temperatura de $97,5^{\circ} \mathrm{C}$ por 40 minutos. Após resfriarem em temperatura ambiente por 20 minutos, as lâminas foram lavadas em água destilada e colocadas em solução tampão salina de fosfato (PBS) com $\mathrm{pH}=7,4$ com TWIN 20 (Sigma código P7949), sendo realizados três banhos de cinco minutos cada. Em seguida, os cortes foram incubados com os anticorpos previamente diluídos em uma solução de PBS-TWIN20+BSA a 0,1\%.

A quantificação das células positivas para o anticorpo anti-Ki67 foi realizada num universo de 1.000 células $^{(19)}$. Os tumores foram classificados em duas categorias: tumores $<5 \%$ e aqueles $\geq 5 \%$ de células positivas. A positividade para o anticorpo antiproteína S-100 e antiactina músculo-específico determinou o imunofenótipo dos tumores, classificando-os como: muscular, neural, duplo ou nulo.

Com relação ao anticorpo anti-p16 $6^{\text {ink4a }}$, foram empregados critérios já utilizados ${ }^{(25,37)} \mathrm{em}$ GIST e tumores de partes moles. A marcação foi considerada de baixa expressão quando o número de células não marcadas foi superior a $20 \%$ do total, e de alta expressão quando o número de células positivas foi superior a $80 \%$ do total, dividindo os casos em duas categorias: baixa expressão e alta expressão do p16 ink4a.

\section{Análise estatística}

Utilizou-se o programa SPSS 13.0 com análises das frequências diretas e univariadas de todos os fatores prognósticos estudados com relação à sobrevida global, empregando-se o teste Kaplan Meier, sendo estabelecido valor de significância $<0,05$.

\section{Resultados}

O grupo de estudo de 42 pacientes foi constituído por 25 mulheres $(59,5 \%)$ e 17 homens (40,5\%), com média de idade de 58,48 $\pm 13,71$, variando entre 24 a 83 anos. Com relação à topografia das neoplasias, 18 situavam-se no estômago (42,9\%), 15 no intestino delgado $(35,7 \%)$, seis no intestino grosso $(14,3 \%)$ e 3 no esôfago $(7,1 \%)$.

O tamanho macroscópico das neoplasias variou de 1,2 a $24 \mathrm{~cm}$ (média $8,08 \pm 5,8$ ), sendo que 16 tumores mediam até $5 \mathrm{~cm}$ no maior eixo $(38,1 \%)$ e 26 foram maiores que $5 \mathrm{~cm}(61,9 \%)$.

Quanto ao índice mitótico, 30 casos apresentavam menos que cinco mitoses/50 CGA $(71,4 \%)$ e os demais (12), mais que cinco mitoses/50 CGA $(28,6 \%)$.

Com relação ao subtipo histológico, 23 tumores $(54,8 \%)$ apresentaram padrão fusiforme, cinco epitelioide $(11,9 \%)$ e 14 mistos (33,3\%). A necrose foi presente em 15 casos $(35,7 \%)$ e ausente em 27 casos $(64,3 \%)$.

Quanto ao grau de risco, os tumores apresentaram um caso de baixíssimo risco (2,4\%), 13 casos de baixo risco (31\%), 13 casos de risco intermediário (31\%) e 15 casos de alto risco $(35,7 \%)$.

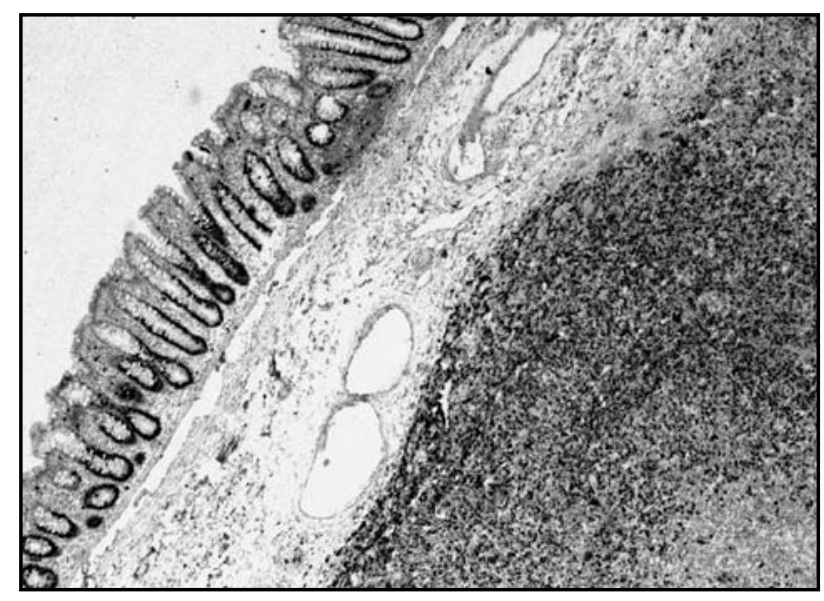

Figura 3 - GIST intestinal. Marcação nuclear imuno-histoquímica (Ki67), fortemente positiva (20X) 
O anticorpo anti-Ki67 mostrou 26 tumores (61,9\%) com índice de proliferação celular menor que $5 \%$, tendo o restante, 16 tumores $(38,1 \%)$, índice maior ou igual a $5 \%$ (Figuras 3 e 4).

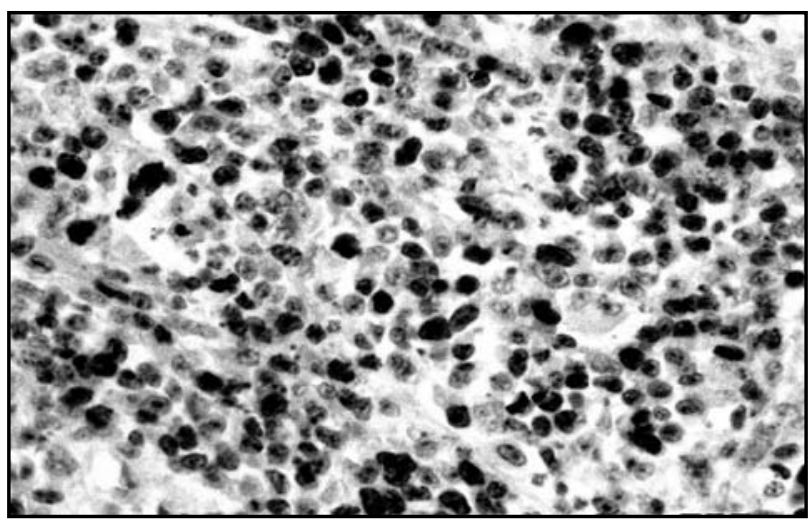

Figura 4 - Em detalhe (40X), marcação das células tumorais maiores que 5\%

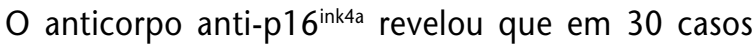
$(71,4 \%)$ a negatividade foi maior ou igual a $20 \%$ e em 12 casos $(28,6 \%)$ a positividade foi maior que $80 \%$.

O imunofenótipo evidenciou 18 tumores com diferenciação muscular (42,9\%), 12 com diferenciação neural (28,6\%), 5 com dupla diferenciação (neural e muscular) $(11,9 \%)$ e 7 tumores com nula diferenciação $(16,7 \%)$.

Quanto ao status dos pacientes, 26 pacientes encontravam-se vivos sem doença $(61,9 \%), 8$ pacientes vivos com doença (19\%) e 8 óbitos em decorrência da doença (19\%), sendo que o período de acompanhamento variou entre um e 195 meses, com mediana de 29 meses.

\section{Valor prognóstico}

A avaliação do valor prognóstico mostrou que as seguintes variáveis estavam associadas a menor sobrevida do paciente: tumores de alto risco, quando comparados com os demais $(p=0,016)$; as variáveis que compõem o grau de risco também mostraram significância estatística quando analisadas isoladamente, ou seja, tumores $>5 \mathrm{~cm}$ $(p=0,017)$, assim como aqueles com índice mitótico superior a cinco mitoses $/ 50 \operatorname{CGA}(p=0,01)$. Outra variável que também se mostrou importante foi a presença de necrose tumoral $(p=0,001)$, além do índice de proliferação celular maior que $5 \%(p<0,001)$. As demais variáveis estudadas não mostraram significância estatística na presente amostra.

\section{Discussão}

A caracterização do comportamento biológico dos GISTs ainda é um dos maiores desafios relacionados com essa neoplasia. Numerosos estudos ${ }^{(2,4,18,19,20,21,25)}$ procuram definir os fatores prognósticos que seriam capazes de estabelecer uma linha divisória entre o tumor estromal de bom prognóstico e aquele de mau prognóstico. Esta revisão confere ao grau de risco importante papel no prognóstico dos pacientes com GIST, a exemplo de outros previamente existentes ${ }^{(9,18-20)}$. Entretanto, observamos que pacientes cuja neoplasia foi classificada como de baixo risco fez-se acompanhar, posteriormente, do desenvolvimento de metástase hepática. Esta situação também já se encontra relatada na literatura, inclusive em casos de baixíssimo risco ${ }^{(9,26)}$. Diante dessas evidências, entende-se que, embora o grau de risco seja um bom indicador do prognóstico do paciente, ele precisa ser mais discutido, principalmente nos tumores classificados como de riscos baixo e intermediário. Destaca-se, nesse contexto, o trabalho de Miettinen et al.(16), em que são propostas mudanças na classificação de risco, com a inclusão da topografia da neoplasia. Esta situação decorre da descoberta de que a localização do tumor influencia no prognóstico, o que não se acreditava anteriormente ${ }^{(9)}$. Sendo assim, neoplasias gástricas teriam prognóstico mais favorável que em outros sítios (intestino delgado, cólons, reto e esôfago), quando semelhantes nos parâmetros morfológicos ${ }^{(16)}$. Entretanto, entende-se que são necessários estudos adicionais aplicando-se o novo sistema em outras séries.

O tamanho macroscópico da lesão $>5 \mathrm{~cm}$ também se constituiu em importante fator prognóstico, pois nesta revisão nenhum paciente com tumor $<5 \mathrm{~cm}$ faleceu em decorrência da doença, embora dois pacientes já apresentassem metástase, diferentemente do grupo de 26 pacientes com tumores maiores que $5 \mathrm{~cm}$, no qual 14 tiveram metástases e/ou faleceram ${ }^{(10,11,15,18,35)}$. Outro critério morfológico que mostrou significância estatística quanto ao prognóstico com relação à sobrevida global foi o índice mitótico. Os tumores com mais de cinco mitoses/50 CGA levaram seus portadores ao óbito e/ou metástase em $83,4 \%$ dos casos. Por outro lado, pacientes com neoplasias que evidenciaram índice mitótico menor que cinco mitoses/50 CGA encontram-se vivos e sem recidiva em $80 \%$ dos casos, achados esses corroborados por outros autores $(10,11,15,18,35)$.

Todavia, devemos atentar para o fato de que estas duas variáveis são aquelas que constituem o grau de risco e que a sua análise de forma isolada deverá ser estudada em uma série maior de casos, para que se possa, assim, verificar o seu real valor prognóstico. Destaca-se por último a necrose, 
por ser o mais significativo fator prognóstico histopatológico encontrado no presente estudo $(p=0,001)$. A necrose foi um achado em 15 tumores, com sete pacientes evoluindo para o óbito (46,7\%), enquanto no grupo sem necrose apenas $1 / 27$ pacientes evoluiu para o óbito (3,7\%).

O Ki67 é um marcador imuno-histoquímico amplamente estudado ${ }^{(2,10,11,19-22,32)}$ e que se mostra de grande utilidade no prognóstico do GIST. Mais recentemente ${ }^{(19,20,35)}$, foi demonstrado que o índice de proliferação maior ou igual a 5\% está relacionado com neoplasias de mau prognóstico e consequente baixa de sobrevida, o que também pode ser constatado neste estudo, pois $87,5 \%$ dos pacientes com tumores cujo índice de proliferação foi $>5 \%$ apresentaram metástase e/ou faleceram, enquanto em casos cujo índice foi $<5 \%$, apenas $7,7 \%$ evidenciaram tal resultado. Entende-se, assim, de acordo com os resultados de outras séries e também da nossa, que o Ki67 tem grande utilidade como fator prognóstico; contudo, acredita-se que ele deve estar associado a outro fator ou a fatores morfológicos e/ou imuno-histoquímicos, na tentativa de otimizar seu emprego, devido à existência de casos de tumores com baixo índice de proliferação, de mau prognóstico, e até neoplasias com alto índice de proliferação, de bom prognóstico.

Existem, ainda, outros estudos na literatura que analisam fatores prognósticos ${ }^{(3,6,28,31,34)}$, porém a dificuldade de compará-los com o atual material e, ao mesmo tempo, com outros estudos mais recentes ${ }^{(10,18,19,35)}$ provém da incerteza de que esses autores estariam revendo casos de verdadeiros GIST, já que somente após 1998, quando Hirota et al. apresentaram o marcador imuno-histoquímico específico, passou-se a ter maior certeza sobre a casuística em discussão. Além disso, algumas revisões ${ }^{(10,28,32)}$ utilizam diferentes metodologias que estão representadas pela forma de contagem do índice mitótico, medição do tamanho macroscópico da lesão tumoral e avaliação da presença ou não de necrose, o que dificulta a comparação e utilização dos resultados dessas análises no que diz respeito à procura por critérios morfológicos prognósticos do GIST.

Quanto ao p16 6inka, não houve correlação com o prognóstico, pois no grupo (30 pacientes) em que este deveria mostrar mau prognóstico (metástase e/ou óbito), há 18 pacientes vivos e sem doença (60\%), adicionado ao fato de que outro conjunto (12 pacientes), que deveria mostrar tumores com bom prognóstico, apresenta quatro casos de mau prognóstico (33,3\%), com os nossos achados corroborados por Nakamura et al.(19). Tal fato pode ter sido influenciado pela diferente metodologia utilizada, pois nos estudos em que houve correlação $(7,25,29)$, a pesquisa foi feita por biologia molecular. De qualquer forma, em nossa opinião, não se deve descartar o marcador p16 $6^{\text {inka }}$, por enquanto, como possível fator prognóstico do GIST, e sim constituir uma série de casos heterogêneos para a sua pertinente indicação.

Quanto à topografia geral das neoplasias estromais, subtipos histológico e imunofenótipo, não houve correlação com a sobrevida global, o que confirma os achados de outras séries ${ }^{(18-20)}$.

\section{Conclusão}

O grau de risco continua sendo importante na avaliação do prognóstico dos pacientes com GIST, contudo, sugerimos que este deve ser empregado associado ao índice de proliferação celular (Ki67) e à presença de necrose, que viria de encontro à caracterização dos grupos de risco, separando assim os pacientes que necessitam de terapia adjuvante imediata daqueles que devem ser acompanhados ambulatorialmente.

\section{Referências}

1. ANDERSSON, J. et al. The complexity of KIT gene mutations and chromosome rearrangements and their clinical correlation in gastrointestinal stromal (pacemaker cell) tumors. Am J Pathol, v. 160, n. 1, p. 15-22, 2002.

2. BUCHER, P. et al. Are there any prognostic factors for small intestinal stromal tumors? Am J Surg, v. 187, n. 6, p. 761-6, 2004

3. CARRILLO, R. et al. Prognostic significance of DNA ploidy and proliferative index (MIB-1 index) in gastrointestinal stromal tumors. Hum Pathol, v. 28, n. 2, p. 160-5, 1997.

4. CHANGCHIEN, C. R. et al. Evaluation of prognosis for malignant rectal gastrointestinal stromal tumor by clinical parameters and immunohistochemical staining.
Dis Colon Rectum, v. 47, n. 11, p. 1922-9, 2004.

5. DEMATTEO, R. P. etal. Clinical management of gastrointestinal stromal tumors: before and after STI-571. Hum Pathol, v. 33, n. 5, p. 466-77, 2002.

6. DOUGHERTY, M. J. et al. Sarcomas of the gastrointestinal tract. Separation into favorable and unfavorable prognostic groups by mitotic count. Ann Surg, v. 214, n. 5, p. 569-74, 1991.

7. EL-RIFAI, W. et al. DNA sequence copy number changes in gastrointestinal stromal tumors: tumor progression and prognostic significance. Cancer Res, v. 60, n. 14, p. 3899-903, 2000.

8. FERCHICHI, L. et al. Extragastrointestinal stromal tumors: a report of 4 cases. Ann Chir, v. 131, n. 4, p. 271-5, 2006. 
9. FLETCHER, C. D. et al. Diagnosis of gastrointestinal stromal tumors: A consensus approach. Hum Pathol, v. 33, n. 5, p. 459-65, 2002

10. FUJIMOTO, Y. et al. Clinicopathologic study of primary malignant gastrointestinal stromal tumor of the stomach, with special reference to prognostic factors: analysis of results in 140 surgically resected patients. Gastric Cancer, v. 6, n. 1, p. 39-48, 2003.

11. HASEGAWA, T. et al. Gastrointestinal stromal tumor: consistent CD117 immunostaining for diagnosis, and prognostic classification based on tumor size and MIB1 grade. Hum Pathol, v. 33, n. 6, p. 669-76, 2002.

12. HEINRICH, M. C. et al. Biology and genetic aspects of gastrointestinal stromal tumors: KIT activation and cytogenetic alterations. Hum Pathol, v. 33, n. 5, p 484-95, 2002

13. HIROTA, S. et al. Gain-of-function mutations of c-kit in human gastrointestinal stromal tumors. Science, v. 279, n. 5350, p. 577-80, 1998.

14. KINDBLOM, L. G. et al. Benign epithelioid schwannoma. Am J Surg Pathol, v. 22, n. 6, p. 762-70, 1998.

15. MIETTINEN, M. et al. Evaluation of malignancy and prognosis of gastrointestinal stromal tumors: a review. Hum Pathol, v. 33, n. 5, p. 478-83, 2002.

16. MIETTINEN, M.; LASOTA, J. Gastrointestinal stromal tumors: pathology and prognosis at different sites. Semin Diagn Pathol, v. 23, n. 2, p. 70-83, 2006. Review.

17. MIETTINEN, M.; SARLOMO-RIKALA, M.; LASOTA, J. Gastrointestinal stromal tumors: recent advances in understanding of their biology. Hum Pathol, v. 30, n. 10, p. 1213-20, 1999

18. MIETTINEN, M.; SOBIN, L. H.; LASOTA, J. Gastrointestinal stromal tumors of the stomach: a clinicopathologic, immunohistochemical, and molecular genetic study of 1765 cases with long-term follow-up. Am J Surg Pathol, v. 29, n. 1, p. 52-68, 2005.

19. NAKAMURA, N. et al. Prognostic significance of expressions of cell-cycle regulatory proteins in gastrointestinal stromal tumor and the relevance of the risk grade. Hum Pathol, v. 36, n. 7, p. 828-37, 2005.

20. NILSSON, B. et al. Gastrointestinal stromal tumors: the incidence, prevalence, clinical course, and prognostication in the preimatinib mesylate era-a population-based study in western Sweden. Cancer, v. 103, n. 4, p. 821-9, 2005.

21. OZGUC, H. et al. Analysis of prognostic and immunohistochemical factors in gastrointestinal stromal tumors with malignant potential. J Gastrointest Surg, v. 9, n. 3, p. 418-29, 2005.

22. PANIZO-SANTOS, A. et al. Predicting Metastatic Risk of Gastrointestinal Stromal Tumors: Role of Cell Proliferation and Cell Cycle Regulatory Proteins. Int J Surg Pathol, v. 8, n. 2, p. 133-44, 2000.
23. PARK, S. S. et al. Malignant extragastrointestinal stromal tumor of retroperitoneum. Acta Oncoli, v. 44, n. 5, p. 497-9, 2005

24. PEREZ-ATAYDE, A. R.; SHAMBERGER, R. C.; KOZAKEWICH, H. W. Neuroectodermal differentiation of the gastrointestinal tumors in the Carney triad. An ultrastructural and immunohistochemical study. Am J Surg Pathol, v. 17, n. 7, p. 706-14, 1993.

25. $\mathrm{RICCl}, \mathrm{R}$. et al. Role of $\mathrm{p} 16^{\text {ink4a/lnk4a }}$ in gastrointestinal stromal tumor progression. Am J Clin Pathol, v. 122, n. 1, p. 35-43, 2004

26. ROSSI, C. R. et al. Gastrointestinal stromal tumors: from a surgical to a molecular approach. Int J Cancer. V. 107, n. 2, p. 171-6, 2003.

27. RUBIN, B. P. et al. KIT activation is a ubiquitous feature of gastrointestinal stromal tumors. Cancer Res, v. 61, n. 22, p. 8118-21, 2001.

28. RUDOLPH, P. et al. Immunophenotype, proliferation, DNA ploidy, and biological behavior of gastrointestinal stromal tumors: a multivariate clinicopathologic study. Hum Pathol, v. 29, n. 8, p. 791-800, 1998.

29. SCHNEIDER-STOCK, R. et al. High prognostic value of p16 ink4alNK4 alterations in gastrointestinal stromal tumors. J Clin Oncol, v. 21, n. 9, p. 1688-97, 2003.

30. SHINOMURA, Y. et al. Pathophysiology, diagnosis, and treatment of gastrointestinal stromal tumors. J Gastroenterol, v. 40, n. 8, p 775-80, 2005.

31. SUSTER, S.; FLETCHER, C. D. Gastrointestinal stromal tumors with prominent signet-ring cell features. Mod Pathol, v. 9, n. 6, p. 609-13, 1996.

32. TORNOCZKY, T.; KOVER, E.; PAJOR, L. Frequent occurrence of low grade cases among metastatic gastrointestinal stromal tumours. J Clin Pathol, v. 56, n. 5, p. 367-7, 2003

33. TRAN, T.; DAVILA, J. A.; EL-SERAG, H. B. The epidemiology of malignant gastrointestinal stromal tumors: an analysis of 1458 cases from 1992 to 2000. Am J Gastroenterol, v. 100, n. 1, p. 162-8, 2005.

34. UEYAMA, T. et al. A clinicopathologic and immunohistochemical study of gastrointestinal stromal tumors. Cancer, v. 69, n. 4, p. 947-55, 1992.

35. WONG, N. A. et al. Prognostic indicators for gastrointestinal stromal tumours: a clinicopathological and immunohistochemical study of 108 resected cases of the stomach. Histopathology, v. 43, n. 2, p. 118-26, 2003

36.YARDEN, Y. et al. Human proto-oncogene c-kit: a new cell surface receptor tyrosine kinase for an unidentified ligand. Embo J, v. 6, n. 11, p. 3341-51, 1987.

37. YOO, J. et al. Expression of E-cadherin and $\mathrm{p} 53$ proteins in human soft tissue sarcomas. Arch Pathol Lab Med, v. 126, n. 1, p. 33-8, 2002
Endereço para correspondência

Rodrigo Panno Basilio de Oliveira

Rua Álvaro Ramos, 71/505

CEP: $22.280-110$ - Rio de laneiro-R

Tel.: (21) 2542-6028 e $9948-4017$ 